\title{
Analysis of Kinetoplast DNA from Mexican Isolates of Leishmania (L.) mexicana
}

\author{
Omar Hernández-Montes, ${ }^{1}$ Saúl González Guzmán, ${ }^{1}$ Federico Martínez Gómez, ${ }^{2}$ \\ Douglas C. Barker, ${ }^{3}$ and Amalia Monroy-Ostria ${ }^{1}$ \\ ${ }^{1}$ Department of Immunology, National School of Biological Sciences, National Polytechnic Institute, 11340 México City, Mexico \\ ${ }^{2}$ Department of Parasitology, National School of Biological Sciences, National Polytechnic Institute, 11340 México City, Mexico \\ ${ }^{3}$ Christ's College, Cambridge CB2 3BU, UK
}

Correspondence should be addressed to Amalia Monroy-Ostria, amaliahmo@gmail.com

Received 1 June 2012; Revised 11 October 2012; Accepted 22 October 2012

Academic Editor: Fabiana Pimenta

Copyright ( $) 2012$ Omar Hernández-Montes et al. This is an open access article distributed under the Creative Commons Attribution License, which permits unrestricted use, distribution, and reproduction in any medium, provided the original work is properly cited.

\begin{abstract}
This study analyzed DNA minicircles of Mexican isolates of L. (Leishmania) mexicana to look for genetic differences between strains isolated from patients with diffuse cutaneous (DCL) and localized (LCL) leishmaniasis. The kDNA was analyzed using polymerase chain reaction (PCR), restriction fragment polymorphism analysis of the PCR products (PCR-RFLP) and the PCR products were sequenced. In the PCR with primers specific for the subgenus Leishmania, the Mexican isolates gave higher amplification products than the other L. mexicana complex strains and with specific primers for the L. mexicana complex they were poorly amplified. In the PCR-RFLP analysis with the Eco RV, Hae III, and Mbo I endonucleases, the Mexican isolates displayed similar restriction patterns, but different from the patterns of the other members of the L. mexicana complex. In the phylogenetic tree constructed, the kDNA sequences of the Mexican clones formed two groups including sequences of LCD or LCL clones, apart from the other $L$. mexicana complex members. These results suggest that the kDNA minicircles of the Mexican isolates are more polymorphic than the kDNA of other members of the L. mexicana complex and have different recognition sites for the restriction enzymes used in this study.
\end{abstract}

\section{Introduction}

Cutaneous leishmaniasis (CL) is the most widespread form of leishmaniasis, causing primary localized skin lesions (LCL) from which parasites can disseminate to the nasopharyngeal mucosa and cause the disfiguring lesions typical of mucocutaneous leishmaniasis (MCL) or disseminated to the entire body as nodular lesions (DCL) [1].

American cutaneous leishmaniasis is characterized by a spectrum of clinical presentations. These include LCL caused by L. (Leishmania) mexicana, DCL caused by L. (Leishmania) amazonensis, L. (Leishmania) venezuelensis, and L. (Leishmania) pifanoi, all belonging to the Leishmania mexicana complex, and MCL caused by members of the Leishmania braziliensis complex [2].

Cutaneous leishmaniasis in Mexico is highly endemic with broad geographical distribution of the different clinical manifestations. In endemic areas, LCL or MCL can be caused by members of both L. mexicana and L. braziliensis complexes [3], making more accurate analysis and identification of the Leishmania strains imperative so that opportune and appropriate treatment can be administered.

Polymerase chain reaction (PCR) approaches have been used in parasite DNA analysis. Several PCR molecular targets have been developed, including minicircle kinetoplast DNA (kDNA) [3], the miniexon (spliced leader RNA) gene [4], and the gp63 PCR-RFLP [5], among others.

Kinetoplast DNA contains approximately 10,000 minicircles per cell that are around 800 base pairs (bp) in size, with a roughly $200 \mathrm{bp}$ conserved region and an approximately $600 \mathrm{bp}$ variable region [6, 7]. The sequence classes of minicircles are organized in a uniform scheme and contain one or several highly conserved regions (CR). There are three highly conserved blocks (CSB) within CRs: CSB1 
(GGGCGT), CSB2 (CCCCGTTC), and CSB3 (GGGGTT GGTGTA) known as the universal minicircle sequence (UMS) $[6,7]$. Minicircles are subjected to frequent insertions and deletions leading to diversity in size; removal and insertion of recognition sites for various restriction enzymes and some size heterogeneity in the overall minicircle size can be observed [8].

In the present study, the analysis of kDNA minicircles of isolates of $L$. (L.) mexicana from skin lesions of patients with DCL or LCL was performed in order to determine whether a particular minicircle class is exclusive of one strain of Leishmania or the differences in clinical manifestation of the disease are related to any particular minicircles classes.

\section{Materials and Methods}

2.1. Leishmania Species and Culture Conditions. This study was reviewed and approved by the Ethics Committee of the Escuela Nacional de Ciencias Biologicas, Instituto Politecnico Nacional, Mexico, in agreement with international ethics guidelines for biomedical research involving human subjects (Ley General de Salud, Mexico).

The study was conducted with seven Mexican isolates of L. (L.) mexicana from patients with multiple nonulcerative nodular skin lesions (DCL) or with ulcerative skin lesions developing at the site of the sandfly bite (LCL) from different states in Mexico (Figure 1), included after informed consent was obtained. For parasite isolation, needle aspirates were taken from the edge of cutaneous lesions prior to patients receiving treatment. The Mexican isolates were sent to the London School for Tropical Medicine and Hygiene (UK) for isoenzyme characterization. Reference strains of members of the L. mexicana complex were also included (Table 1).

The strains of Leishmania were cultured in RPMI medium supplemented with $10 \%$ fetal calf serum at $28^{\circ} \mathrm{C}$.

2.2. DNA Extraction. DNA was prepared, as described elsewhere [3], by centrifuging $10^{9}$ parasites of an exponential phase of growth culture at $1900 \mathrm{~g}$ for $10 \mathrm{~min}$. The pellet was resuspended in $1 \mathrm{~mL}$ of NET 100 (100 mMTris-HCl, pH 8.0; $100 \mathrm{mM}$ EDTA; $100 \mathrm{mM} \mathrm{NaCl}), 1 \%$ SDS, and $4 \mu \mathrm{L}$ of $10 \mathrm{mg} / \mathrm{mL}$ proteinase $\mathrm{K}$ (Sigma) and incubated at $37^{\circ} \mathrm{C}$ overnight, followed by two phenol-chloroform extractions and ethanol precipitation. The DNA extracted was washed with $70 \%$ ethanol and dissolved in $100 \mu \mathrm{L}$ distilled water and kept at $-70^{\circ} \mathrm{C}$ until use.

2.3. Polymerase Chain Reaction. The universal primers AJS1 (GGGGTTGGTGTAAAATAG) and DeB8 (CCAGTTTCC CGCCCCG), specific for kDNA minicircles of the Leishmania subgenus [9], and the M1 (CCAGTTTCGAGCCCC GGAG) and M2 (GGTGTAAAATAGGGCCGGATGCTC) primers, specific for minicircles of the L. mexicana complex [10] were used to amplify kDNA from reference strains and from Mexican isolates. PCR amplifications were done as described elsewhere $[9,10]$, in a solution of $0.2 \mathrm{mM}$ each of deoxyribonucleotide (Invitrogen Life Technologies, Carlsbad, CA, USA), 50 pmol of each specific primer, 2.5

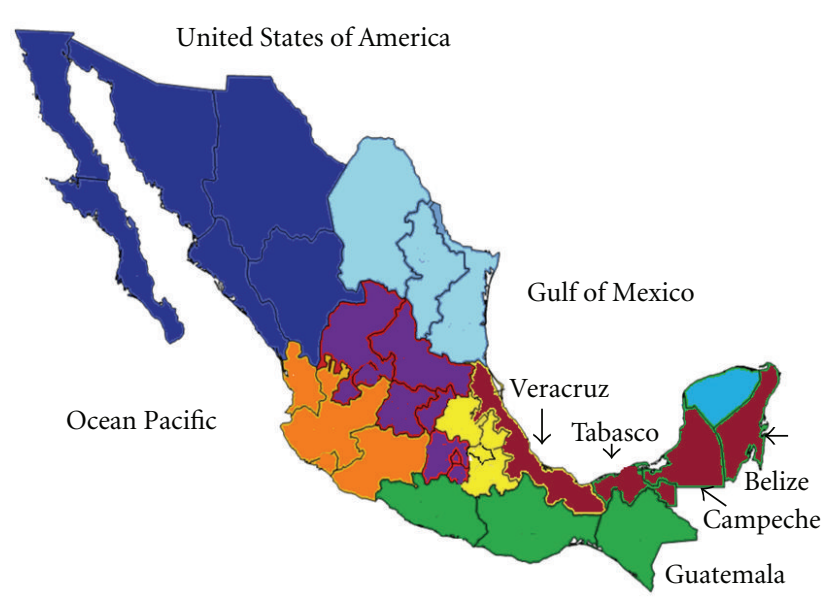

FIGURE 1: Map of Mexico, showing the endemic regions studied in this work, Veracruz, Tabasco, and Campeche states.

units of Taq DNA polymerase (Perkin Elmer Cetus), $100 \mathrm{ng}$ of DNA template, $1.5 \mathrm{mM}$ of $\mathrm{MgCl}_{2}$ in a final volume of $100 \mu \mathrm{L}$. Samples were denatured at $96^{\circ} \mathrm{C}$ for $6 \mathrm{~min}$. PCR (35 cycles) consisted of annealing at $60^{\circ} \mathrm{C}$ for AJS1 and DeB8 primers and $67.5^{\circ} \mathrm{C}$ for $\mathrm{M} 1$ and $\mathrm{M} 2$ primers, extension at $72^{\circ} \mathrm{C}$ for $1 \mathrm{~min}$, and final extension at $72^{\circ} \mathrm{C}$ for $10 \mathrm{~min}$ on a Perkin-Elmer Model 2400 thermal cycler (Perkin Elmer, Roche Molecular Systems Inc., Brancbourg, NJ, USA). PCR products $(10 \mu \mathrm{L})$ were fractionated by electrophoresis in $1 \%$ agarose or $8 \%$ acrylamide gels in TBE (90 mMTris$\mathrm{HCl} \mathrm{pH} \mathrm{8.3,} 90 \mathrm{mM}$ boric acid, and $2 \mathrm{mM}$ EDTA) stained with ethidium bromide $(50 \mathrm{~g} / \mathrm{mL})$ and were observed under a transilluminator (SIGMA Chemical Co., St. Louis, MO, USA).

2.4. Restriction Fragment Polymorphism Analysis of kDNA. The kDNA amplicons obtained with the AJS1 and DeB8 primers of the Leishmania reference strains and the Mexican isolates were digested with the Bam HI, Eco RV, Hae III, Hind III, Kpn I, Mbo I, Mse I, Msp I, and Xba I endonucleases (Gibco BRL). The restrictions were performed following the supplier's instructions, briefly: $10 \mu \mathrm{g}$ of PCR product, $2.5 \mu \mathrm{L}$ of the correspondent buffer, and $10 \mathrm{U}$ of the endonuclease in a final volume of $25 \mu \mathrm{L}$ were incubated at $37^{\circ} \mathrm{C}$ for $2 \mathrm{~h}$; the Taq I was incubated at $67^{\circ} \mathrm{C}$. The reactions were analyzed by electrophoresis as mentioned above.

2.5. Sequencing of Minicircles of Mexican Isolates. The kDNA amplicons of the Mexican isolates, MC (from patient with LCL), and GS (from patient with DCL) obtained with the AJS1 and DeB8 primers were gel purified using a QIA quick gel extraction Kit (Qiagen, Germany), ligated into a TA Cloning vector (pCR II Vector) (Invitrogen Life Technologies), and transformed into Escherichia coli INV $\alpha \mathrm{F}^{\prime}$. Plasmid DNA was extracted using the Wizard Plus Minipreps DNA Purification System (Promega Corporation, Madison, WI, USA) and sequenced. Nucleotide sequences were determined using a dideoxynucleotide chain termination sequence kit 
TABLe 1: Mexican isolates and reference strains of Leishmania mexicana complex used in this study.

(a)

\begin{tabular}{|c|c|c|}
\hline Reference strains & International code $^{1}$ & Source \\
\hline L. (L.) mexicana & MHOM/BZ/62/BEL21 & 1 \\
\hline L. (L.) mexicana & MHOM/BZ/62/M379 & 1 \\
\hline L. (L.) amazonensis & IFLA/BR/67/PH8 & 1 \\
\hline L. (L.) amazonensis & MHOM/BR/73/M2269 & 1 \\
\hline L. (L.) garnhami & MHOM/VE/75/HM76 & 1 \\
\hline L. (L.) garnhami & MHOM/VE/76/JAP78 & 1 \\
\hline L. (L.) pifanoi & MHOM/VE/57/LL1 & 1 \\
\hline
\end{tabular}

${ }^{1}$ : The WHO Leishmania Reference Collection at the London School of Hygiene and Tropical Medicine, London, UK.

(b)

\begin{tabular}{|c|c|c|}
\hline & Mexican isolates $^{2}$ & Origin \\
\hline \multicolumn{3}{|c|}{ From patients with LCL } \\
\hline L. (L.) mexicana & MHOM/MX/88/HRC JS (SOB) & Tabasco \\
\hline L. (L.) mexicana & MHOM/MX/88/HRC MC (MC) & Tabasco \\
\hline L. (L.) mexicana & MHOM/MX/83/UAYV (YUC) & Campeche \\
\hline \multicolumn{3}{|c|}{ From patients with DCL } \\
\hline L. (L.) mexicana & MHOM/MX/84/ISET GS (GS) & Tabasco \\
\hline L. (L.) mexicana & MHOM/MX/85/ISET HF (HF) & Veracruz \\
\hline L. (L.) Mexicana & MHOM/MX/92/INDRE AM (AM) & Veracruz \\
\hline L. (L.) mexicana & MHOM/MX/92/INDRE AG (AG) & Tabasco \\
\hline
\end{tabular}

${ }^{2}$ : Instituto Nacional de Diagnostico y Referencia Epidemiologicos, Secretaria de Salud, Mexico. Tabasco, Veracruz, and Campeche states are located in the Mexican Southeast.

(ABI PRISM Dye Terminator Cyclers Sequencing Ready Reaction Kit, Perkin Elmer) and an Abi Prism M 310 Genetic Analyzer automated sequencer (Perkin Elmer).

2.6. Sequence Alignments And Phylogenetic Analysis. The sequences were edited with the DNAMAN, Chromas version 2.0, and Seaview software [11]. Multiple sequences were aligned using Clustal-X Ver. 1.83 [12]. An unrooted phylogenetic tree was constructed using the neighbor-joining method [13] with the Clustal-X program. Evolutionary distances were calculated using Kimura's two-parameter method [14], with MEGA (Molecular Evolutionary Genetics Analysis), Version 3.1. [15]. A total of 1000 bootstrapping replicates were used for the neighbor-joining analysis to obtain relative support for internal nodes. The kDNA sequence data were compared with the sequences of the other members of the L. mexicana complex previously published in GenBank.

\section{Results}

PCR specific for the Leishmania subgenus performed with the AJS1 and DeB8 primers resulted in the amplification of the kDNA of $L$. (L.) mexicana BEL21 and L. (L.) mexicana M379, the reference strains, and the Mexican isolates (lanes 6-12) giving 700 to $870 \mathrm{bp}$ amplification bands; L. (L.) amazonensis M2269 and PH8 as well as L. (L.) garnhami HM76 and JAP78 gave bands less than 700 bp (Figure 2).
PCR specific for the L. mexicana complex, with the primers M1 and M2, resulted in the amplification of the $L$. (L.) amazonensis reference strain, giving $700 \mathrm{bp}$ amplification bands; the other references strains gave 800 bp bands. The Mexican isolates were poorly amplified, giving $680 \mathrm{bp}$ bands (data not shown).

Restriction length polymorphism analysis. In endonuclease digestion of kDNA amplicons with the Eco RV endonuclease, the Mexican isolates displayed a six band pattern (lanes 6-12), the reference strain L. (L.) mexicana Bel21 showed eight bands (lane 5), the other Leishmania strains showed two bands and some were not restricted (Figure 3). With Hae III the Mexican isolates all displayed the same pattern, which was several bands in length, the L. mexicana complex reference strains also displayed a restriction pattern of several bands, but a different size (data not shown). With Mbo I, all the Mexican isolates displayed a similar twoband restriction pattern (lanes 6-12) and the Leishmania reference strains showed a pattern of two or four bands (Figure 4). With the Xba I only, the Mexican isolates were restricted to displaying patterns several bands long (data not shown); the Hind III, MspI, and Spe I endonucleases restricted only certain strains, but not the Mexican isolates (data not shown). The Bam HI and Kpn I endonucleases did not digest any Leishmania strains (data not shown).

In the cloning and sequencing of $\mathrm{kDNA}$ minicircles of the Mexican isolates, seven minicircle clone sequences were obtained, five from the LCL isolated and two from the DCL (data not shown). All seven clones showed high 


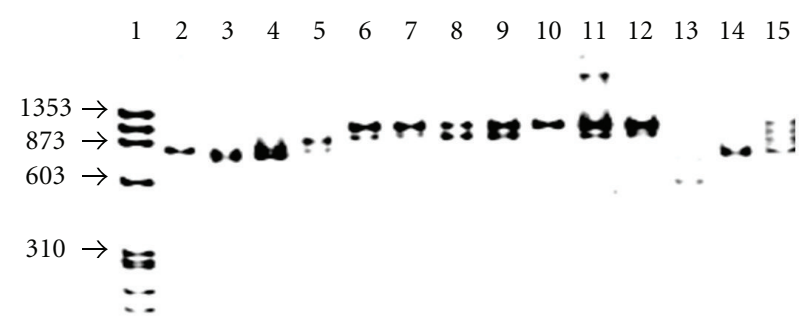

FIgURe 2: PCR Amplification of kDNA with the primers AJS 1 and DeB 8. Lane 1, MWMX174 Hae III; 2, L. (L.) granhami JAP78; 3, L. (L.) granhami HM76; 4, L. (L.) mexicana M379; 5, L. (L) mexicana BEL $21 ; 6, L$. (L) mexicana SOB; 7, L. (L.) mexicana YUC; 8, L. (L.) mexicana MC; 9, L. (L.) mexicana GS; 10, L. (L.) mexicana HF; 11, L. (L.) mexicana AM; 12, L. (L.) mexicana AG; 13, L. (L.) amazonensis M2269; 14, L. (L.) amazonensis PH8; 15, L. (L.) pifanoi L11.

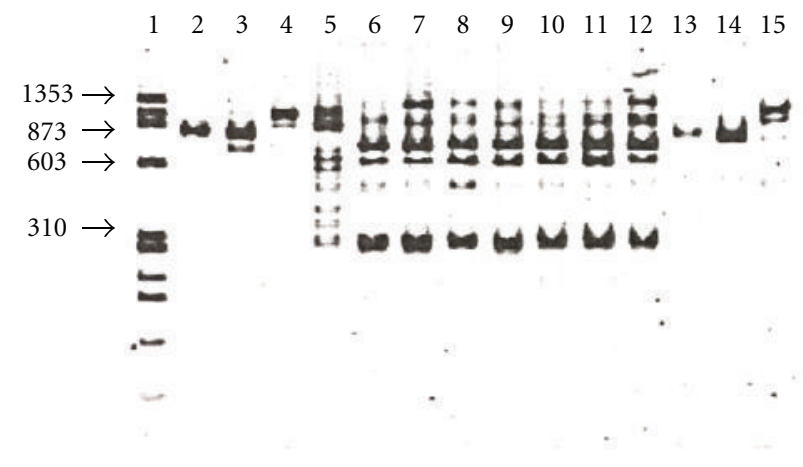

FIgURe 3: PCR-RFLP analysis with the endonuclease Eco RV. Lane 1, MW X174 Hae III; 2, L. (L.) garnhami JAP78; 3, L. (L.) garnhami HM76; 4, L. (L.) mexicana M379; 5, L. (L.) mexicana BEL 21; 6, L. (L.) mexicana SOB; $7, L$. (L.) mexicana YUC; $8, L$. (L.) mexicana MC; 9, L. (L.) mexicana GS; 10, L. (L.) mexicana HF; 11, L. (L.) mexicana $\mathrm{AM} ; 12$, L. (L.) mexicana $\mathrm{AG} ; 13$, L. (L.) amazonensis M2269; 14, L. (L.) amazonensis PH8; 15, L. (L.) pifanoi LL1.

homology in their conserved part, presenting the highly conserved block CSB3 (GGGGTTGGTGTA) known as the universal minicircle sequence (UMS) (Shlomai, 2004), but after position 19 two groups of minicircle sequences have been found, one 757-759 bp (LCL6, LCL14, and LCD15), which presents the ACTCCTGGATTT motif, and the 790 to 791 bp group (LCD7, LCL17, LCL5, and LCL4), which presents the TATCCTCTCCT motif. The comparison of the sequences of these two groups revealed differences in deletions and substitutions of bp along the kDNA minicircle (Figure 5).

Based on sequence alignments of kDNA minicircles of Mexican isolates and the other members of the L. mexicana complex, phylogenetic trees were constructed. The consensus tree showed nine groupings. The first and second groups were formed with LCL and DCL Mexican clones. A further

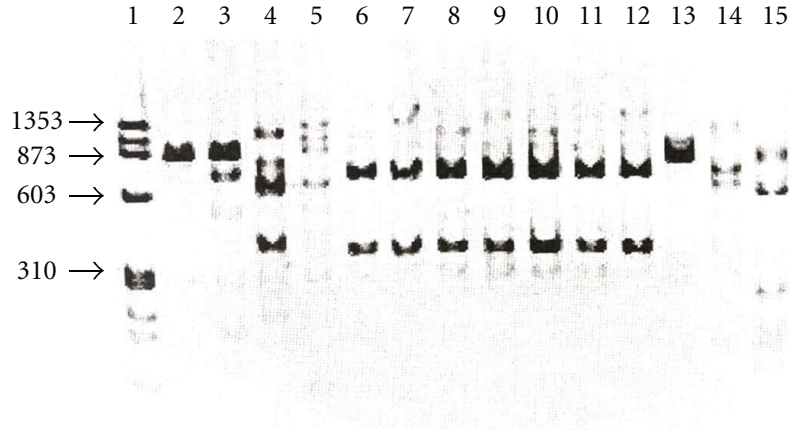

FIgURE 4: PCR-RFLP analysis with the endonuclease Mbo I. Lane 1, MW X174 Hae III; 2, L. (L.) garnhami JAP78; 3, L. (L.) garnhami HM76; 4, L. (L.) mexicana M379; 5, L. (L.) mexicana BEL 21; 6, L. (L.) mexicana SOB; 7, L. (L.) mexicana YUC; 8, L. (L.) mexicana MC; 9, L. (L.) mexicana GS; 10, L. (L.) mexicana HF; 11; L. (L.) mexicana AM; 12, L. (L.) mexicana AG; 13, L. (L.) amazonensis M2269; 14, L. (L.) amazonensis PH8; 15, L. (L.) pifanoi LL1.

two groups were formed with clones of $L$. (L.) mexicana from international strains. Another two groups were formed with $L$. (L.) amazonensis and L. (L.) mexicana clones. Another node was formed with two groups including only $L$. $(L$.) amazonensis clones. The last group was formed with clones of L. (L.) mexicana (Figure 6).

\section{Discussion}

In the amplification of kDNA with the primers specific for the Leishmania subgenus, the Mexican isolates showed PCR products that had a slightly higher molecular weight than the L. (L.) Mexicana M379, L. (L.) mexicana Bel21, $L$. (L.) amazonensis, and $L$. (L.) pifanoi reference strain PCR products (Figure 2). On the other hand, with the specific primers for the L. mexicana complex none of the Mexican isolates were properly amplified as the other members of the L. mexicana complex had been (data not shown). These results could be due to the fact that the specific primers for Leishmania subgenus have the UMS sequence as do the Mexican isolates, and the sequences of the M1 and M2 primers have less homology with the Mexican isolates than with the other members of the L. mexicana complex, indicating that the $\mathrm{kDNA}$ minicircles of the Mexican isolates are different from the other members of the L. mexicana complex.

Interestingly, the Mexican isolates digested with the Eco RV (Figure 3), Hae III (data not shown), and Mbo I endonucleases (Figure 4) displayed similar restriction patterns between them, totally different from the digestion patterns displayed for the other members of the L. mexicana complex. Furthermore, with the Xba I endonuclease only the Mexican isolates were restricted (data not shown). These results clearly suggest that the kDNA minicircles of the Mexican isolates are more polymorphic than the kDNA of the other members of the L. mexicana complex and have different recognition sites for the several restriction enzymes 


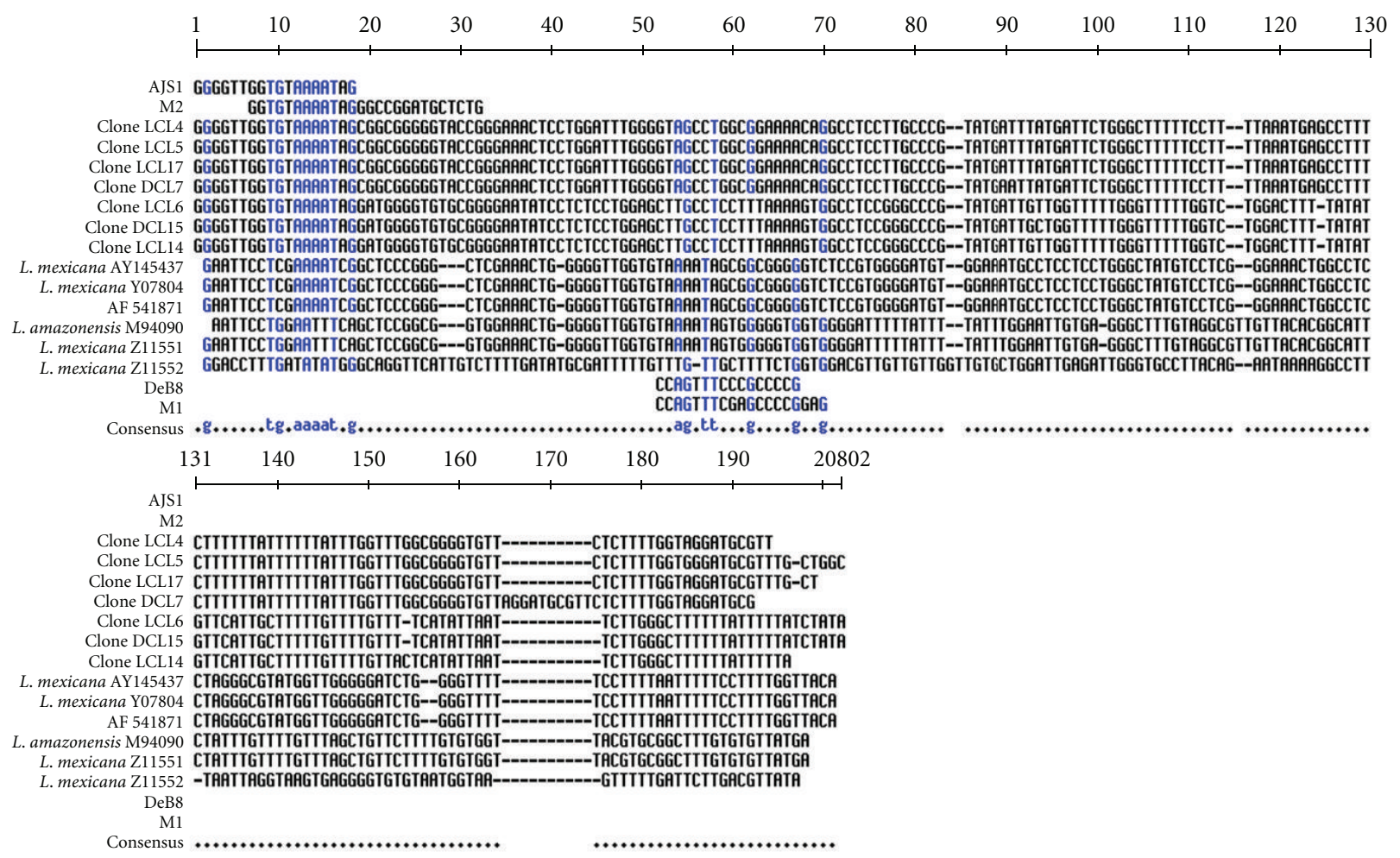

Figure 5: Aligment of the kDNA minicircles sequences classes (from 1 to $200 \mathrm{bp}$ ). Clones of the Mexican isolates: LCL4, DCL7, LCL17, DCL15, LCL6, and LCL14. Sequences of other members of the Leishmania mexicana complex published in the GeneBank: L. mexicanaY07807, AF54187, Y145437; L. (L.) amazonensis M 94090; L. (L.) mexicana Z11551, Z11552, Z11554, Z11556, and Z11549; L. (L.) amazonensis M21325, M 21327; L. (L.) mexicana Z11553, Z11555.

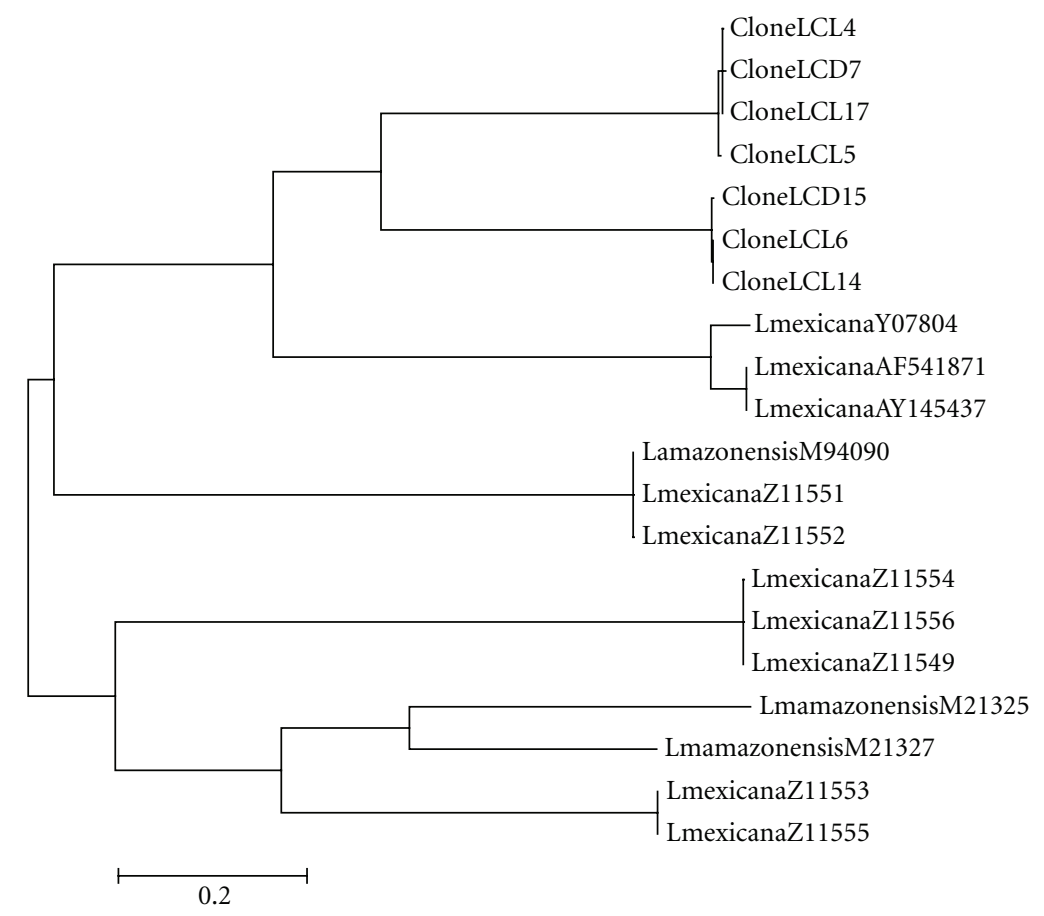

Figure 6: Phylogenetic tree constructed with sequences of kDNA minicircles. Leishmania mexicana complex members published in theGenBank: L. (L.) mexicana Y07807, AF54187, Y145437; L. (L.) amazonensis M 94090; L. (L.) mexicana Z11551, Z11552, Z11554, Z11556, and Z11549; L. (L.) amazonensis M21325, M 21327; L. (L.) mexicana Z11553, Z11555; clones of L. (L.) mexicana. Mexican isolates clones: LCL4, DCL7, LCL17, LCL5, DCL15, LCL6, and LCL14. 
used in this study. Furthermore, in studies of PRC-RFLP of kDNA [16] found six basic digestion patterns in Mexican isolates of $L$. (L.) mexicana that were different from the digestion patterns displayed for the other members of the $L$. mexicana complex reference strains.

The Mexican isolate clone sequence classes of minicircles formed two groups including DCL and LCL clones in each group, indicating that a particular minicircle class is not exclusive of one strain of Leishmania, furthermore the different clinical manifestation of the disease (LCL or DCL) is not related to any particular minicircle classes (Figure 5). Comparing these sequences with the sequences of other members of the L. mexicana complex, it was observed that they did not have extensive sequence homology. These results agree with Barker's studies [17] showing that the minicircles of representative strains of the main complexes of Leishmania do not share extensive sequence homology.

In the phylogenetic tree constructed with these sequences, the other members of the L. mexicana complex formed groups with $L$. (L.) mexicana and $L$. (L.) amazonensis clones apart from the groups formed exclusively with the Mexican clones. These may indicate that the Mexican isolates have sequence classes of minicircles that are different from the other members of the L. mexicana complex isolated in other countries (Figure 6).

On the other hand, Gutiérrez-Solar et al. [18] found greater homology between minicircles of geographically separated isolates than minicircles of the same stock. Within human species of Leishmania, Rogers and Wirth [19] described highly homogeneous sequences only in minicircles of geographically related isolates.

On the other hand, in studies with $L$. (L.) amazonensis resistant to tunicamycin, Lee et al. [20] found that certain minor minicircle sequence were selected to replicate and to replace the dominant type of minicircles and become predominant.

Although Leishmania kDNA is very polymorphic, the PCR of kDNA analysis is a very useful and sensitive enough for detecting $L$. donovani genes in skin biopsy specimens from patients and is recommended as a confirmatory diagnostic tool for PKDL [21], and for characterization of cutaneous isolates of Leishmania by isoenzyme typing combined with kDNA restriction analysis [22].

In conclusion it seems that the minicircle classes could change in the kDNA network [8]. We cannot say that one or another minicircle sequence class within the kDNA network is permanent or typical of a virulent or less virulent Leishmania strain, and the clinical manifestation of the disease (LCL or DCL) is not related to any particular minicircle classes. Furthermore there is no relation with their geographical distribution.

\section{Acknowledgments}

This work received the financial support of Secretaria de Investigacion y Postgrado, Instituto Politecnico Nacional, Mexico. A. Monroy-Ostria and F. Martínez-Gómez are sponsored by CONACyT, EDI and COFAA IPN, Mexico.

\section{References}

[1] R. Reithinger, J. C. Dujardin, H. Louzir, C. Pirmez, B. Alexander, and S. Brooker, "Cutaneous leishmaniasis," The Lancet Infectious Diseases, vol. 7, no. 9, pp. 581-596, 2007.

[2] R. Lainson, "The American leishmaniasis: some observations on their ecology and epidemiology," Transactions of the Royal Society of Tropical Medicine and Hygiene, vol. 77, no. 5, pp. 569-596, 1983.

[3] O. Hernández-Montes, A. Monroy-Ostria, S. McCann, and D. C. Barker, "Identification of Mexican Leishmania species by analysis of PCR amplified DNA," Acta Tropica, vol. 71, no. 2, pp. 139-153, 1998.

[4] O. Fernandes, V. K. Murthy, U. Kurath, W. M. Degrave, and D. A. Campbell, "Mini-exon gene variation in human pathogenic Leishmania species," Molecular and Biochemical Parasitology, vol. 66, no. 2, pp. 261-271, 1994.

[5] K. Victoir, S. De Doncker, L. Cabrera et al., "Direct identification of Leishmania species in biopsies from patients with American tegumentary leishmaniasis," Transactions of the Royal Society of Tropical Medicine and Hygiene, vol. 97, no. 1, pp. 80-87, 2003.

[6] D. S. Ray, "Conserved sequence blocks in kinetoplast minicircles from diverse species of trypanosomes," Molecular and Cellular Biology, vol. 9, no. 3, pp. 1365-1367, 1989.

[7] J. Shlomai, "The structure and replication of kinetoplast DNA," Current Molecular Medicine, vol. 4, no. 6, pp. 623-647, 2004.

[8] N. Singh, M. D. Curran, D. Middleton, and A. K. Rastogi, "Characterization of kinetoplast DNA minicircles of an Indian isolate of Leishmania donovani," Acta Tropica, vol. 73, no. 3, pp. 313-319, 1999.

[9] A. J. Smyth, A. Ghosh, M. Q. Hassan et al., "Rapid and sensitive detection of Leishmania kinetoplast DNA from spleen and blood samples of kala-azar patients," Parasitology, vol. 105, no. 2, pp. 183-192, 1992.

[10] S. Eresh, S. M. McCallum, and D. C. Barker, "Identification and diagnosis of Leishmania mexicana complex isolates by polymerase chain reaction," Parasitology, vol. 109, no. 4, pp. 423-433, 1994.

[11] N. Galtier, M. Gouy, and C. Gautier, "SEAVIEW and PHYLO_WIN: two graphic tools for sequence alignment and molecular phylogeny," Computer Applications in the Biosciences, vol. 12, no. 6, pp. 543-548, 1996.

[12] J. D. Thompson, T. J. Gibson, F. Plewniak, F. Jeanmougin, and D. G. Higgins, "The CLUSTAL X windows interface: flexible strategies for multiple sequence alignment aided by quality analysis tools," Nucleic Acids Research, vol. 25, no. 24, pp. 4876-4882, 1997.

[13] N. Saitou and M. Nei, "The neighbor-joining method: a new method for reconstructing phylogenetic trees." Molecular Biology and Evolution, vol. 4, no. 4, pp. 406-425, 1987.

[14] M. Kimura, "A simple method for estimating evolutionary rates of base substitutions through comparative studies of nucleotide sequences," Journal of Molecular Evolution, vol. 16, no. 2, pp. 111-120, 1980.

[15] A. D. Baxavanis and B. F. Ouellette, "Phylogenetic analysis," in Bioinformatics. A Practical Guide to the Analysis of Genes and Proteins, pp. 323-343, Wiley Interscience, New York, NY, USA, 2001.

[16] M. Berzunza-Cruz, G. Bricaire, S. Z. Romero et al., "Leishmania mexicana mexicana: genetic heterogeneity of Mexican isolates revealed by restriction length polymorphism analysis 
of kinetoplast DNA," Experimental Parasitology, vol. 95, no. 4, pp. 277-284, 2000.

[17] D. C. Barker, "DNA diagnosis of human leishmaniasis," Parasitology Today, vol. 3, no. 6, pp. 177-184, 1987.

[18] B. Gutiérrez-Solar, A. J. Smyth, J. Alvar, and D. C. Barker, "Leishmania infantum: sequence homology within minicircle classes regardless of geographical distance," Experimental Parasitology, vol. 81, no. 3, pp. 416-419, 1995.

[19] W. O. Rogers and D. F. Wirth, "Generation of sequence diversity in the kinetoplast DNA minicircles of Leishmania mexicana amazonensis," Molecular and Biochemical Parasitology, vol. 30, no. 1, pp. 1-8, 1988.

[20] S. T. Lee, H. Y. Liu, S. P. Lee, and C. Tarn, "Selection for arsenite resistance causes reversible changes in minicircle composition and kinetoplast organization in Leishmania mexicana," Molecular and Cellular Biology, vol. 14, no. 1, pp. 587-596, 1994.

[21] S. A. Nasreen, A. Hossain, S. K. Paul et al., "PCR-based detection of Leishmania DNA in skin samples of post kalaazar dermal leishmaniasis patients from an endemic area of Bangladesh," Japanese Journal of Infectious Diseases, vol. 65, no. 4, pp. 315-317, 2012.

[22] J. Urbano, M. E. Sanchez-Moreno, C. E. Ovalle et al., "Characterization of cutaneous isolates of Leishmania in Colombia by isoenzyme typing and kDNA restriction analysis," Revista Ibero-Latinoamericana de Parasitología, vol. 70, pp. 16-24, 2011. 


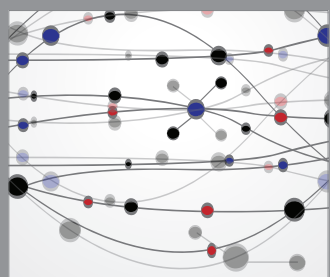

The Scientific World Journal
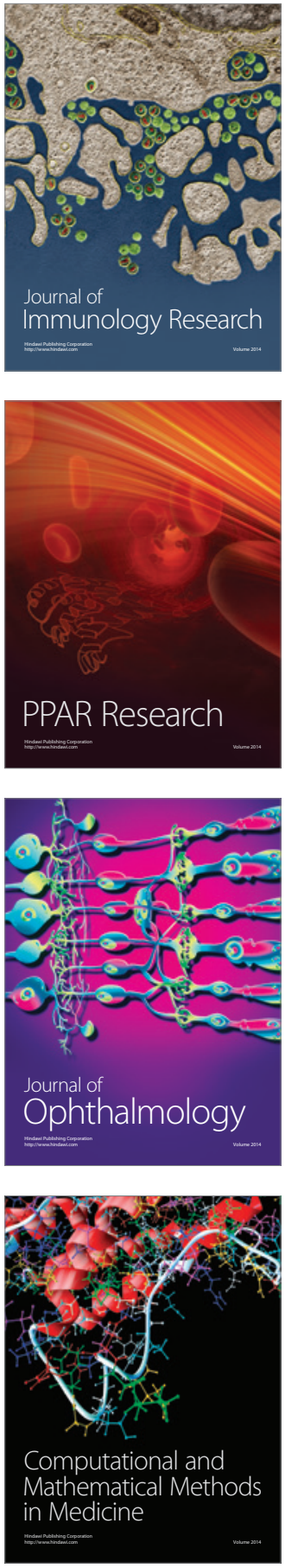

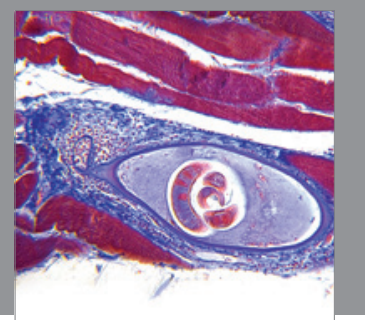

Gastroenterology

Research and Practice
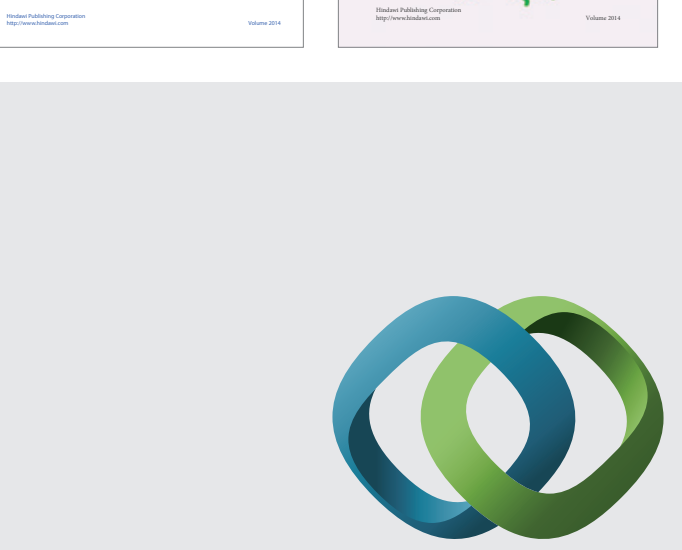

\section{Hindawi}

Submit your manuscripts at

http://www.hindawi.com
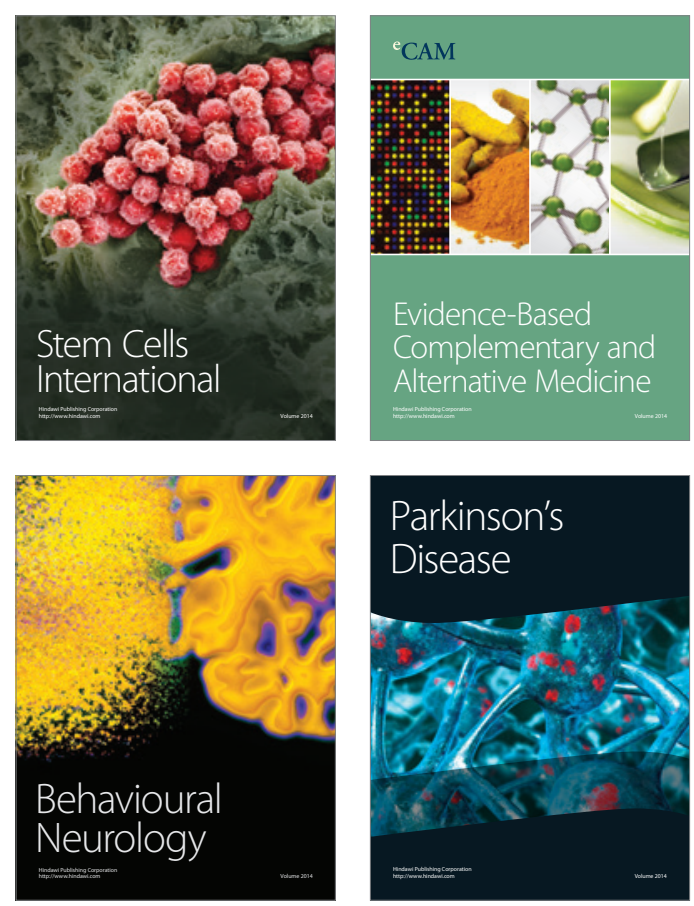

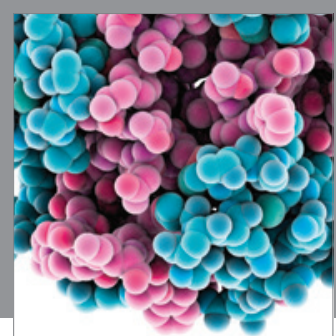

Journal of
Diabetes Research

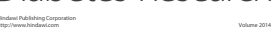

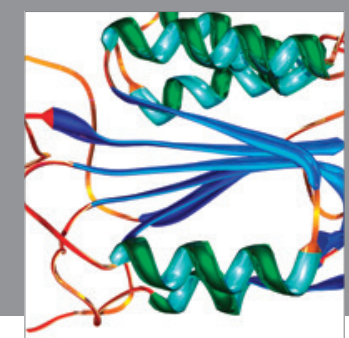

Disease Markers
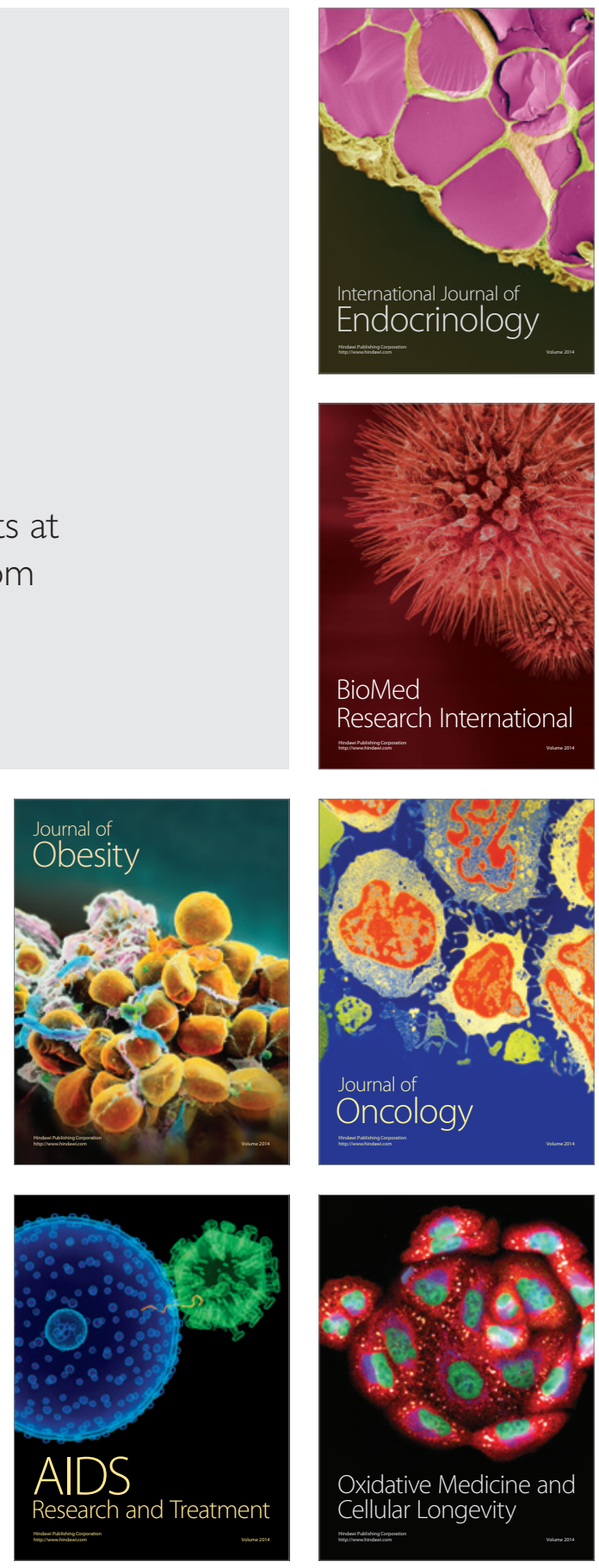